\title{
Understanding burden in caregivers of colorectal cancer survivors: what role do patient and caregiver factors play?
}

\author{
R. Maguire BA (Hons), MA, PhD Lecturer ${ }^{1}$ | P. Hanly BA (Hons), MA, PhD Lecturer ${ }^{1}$ | \\ P. Hyland BA (Hons), MA, PhD Lecturer ${ }^{1}$ | L. Sharp BSc, MSc, PhD Professor ${ }^{2}$
}

${ }^{1}$ National College of Ireland, Dublin, Ireland

${ }^{2}$ Newcastle University, Newcastle upon Tyne, UK

Correspondence

Rebecca Maguire, National College of Ireland, Dublin, Ireland.

Email: rebecca.maguire@ncirl.ie
This study investigated how both caregiver and patient factors predict different aspects of burden in colorectal cancer caregivers. One hundred and fifty-three caregiver-survivor dyads separately provided information on patient disease and treatment-related factors, and perceived global health status (EORTC QLQ30), along with caregiver socio-demographic factors, health and care-related activities. Four multiple regression analyses were conducted to assess the influence of caregiver characteristics, patient characteristics and care-related activities on four dimensions of burden from the Caregiver Reaction Assessment scale. Caregiver characteristics significantly predicted health and financial burden (11\%-13\% of explained variance) with comorbidity and younger age increasing this risk. Patient health, in particular global health status and the presence of a stoma, predicted all burden scores, explaining $14 \%-22 \%$ of variance. Care-related activities was also a significant predictor of all burden scores, explaining an additional $5 \%-11 \%$ of variance, with time involved in caring the most consistent predictor. Results highlight that a combination of factors influence caregiver burden. These results may be used to identify those most at risk, allowing practitioners to deliver tailored effective support. In particular, efforts to alleviate the burden of caring on caregiver schedule may be merited, given that this was the domain in which the burden was greatest.

\section{KEYWORDS}

caregiver burden, caregivers, colorectal cancer, cost of caring, Ireland, oncology

\section{1 | INTRODUCTION}

Cases of colorectal cancer have been increasing internationally along with an increase in survival rates (Bray, Ren, Masuyer, \& Ferlay, 2013). A diagnosis of colorectal cancer has a considerable impact, not only on the patients themselves (Arndt, Merx, Stegmaier, Ziegler, \& Brenner, 2006; Jansen, Koch, Brenner, \& Arndt, 2010) but also on their family members and friends (Cotrim \& Pereira, 2008; Sjovall, Gunnars, Olsson, $\&$ Thomé, 2012). In addition to providing social support, these family members and friends are often required to take on the role of informal carers (hereafter referred to as caregivers), a circumstance which is becoming increasingly common in recent years (Romito, Goldzweig, Cormio, Hagedoorn, \& Andersen, 2013). It is now recognised that in order to support cancer survivors effectively it is vital that the needs of their caregivers are also acknowledged and subsequently met (Girgis et al., 2013a; Olson, 2012; Romito et al., 2013).

Informal cancer caregivers are at an increased risk of stress, anxiety and depression (Olson, 2012); may suffer degradations in their physical health (Sjovall et al., 2011); and may experience detriments in numerous aspects of well-being and quality-of-life (Goren, Gilloteau, Lees, \& daCosta DiBonaventura, 2014; Hanly, Maguire, Hyland, \& Sharp, 2015) with such negative effects persisting long after the diagnosis of the care recipient (Girgis et al., 2013a). These effects have primarily been attributed to the perceived burden of caregiving (Adelman, Tmanova, Delgado, Dion, \& Lachs 2014; Applebaum \& Breitbart, 2013; Girgis, Lambert, Johnson, Waller, \& Currow, 2013b; Romito et al., 2013). Cancer caregivers must take diverse roles, ranging from medical and instrumental, to social and emotional (McMullen et al., 
2014). This can involve substantial time commitments and disruptions to schedule (Yabroff \& Kim, 2009), impose a considerable financial burden (Grov, Fosså, Sørebø, \& Dahl, 2006), and may be further compounded by a perceived lack of social support (Rodakowski, Skidmore, Rogers, \& Schulz, 2012; Yoon, Kim, Jung, Kim, \& Kim, 2014). A greater understanding of the influences on these various aspects of burden in caregivers of cancer survivors in general, and colorectal cancer survivors in particular, is merited given the considerable impact this has on both their physical and mental health (Hanly et al., 2015).

The degree to which caregiver burden is experienced depends on a number of factors which can be attributed to both caregiver and patient characteristics. Identified predictors include caregiver demographic factors such as age, gender and relationship with patient, as well as caregiver health status (Adelman et al., 2014). Patient health status is also a strong predictor, with the severity of symptoms recognised as the key determinant of caregiver burden across a variety of diseases (Brouwer et al., 2004; Forbes, While, \& Mathes, 2007; Sautter et al., 2014). Given the range of adverse physical effects associated with colorectal cancer and its treatment, such as weight loss, nausea, constipation, diarrhoea, pain, dyspnoea, insomnia and fatigue (Arndt et al., 2006), and the presence of a stoma in many cases, caregiver burden may be particularly high in this group (Cotrim \& Pereira, 2008; Ohlsson-Nevo, Andershed, Nilsson, \& Anderzén-Carlsson, 2012).

Given the broad range of factors that potentially contribute to the multidimensional cancer caregiver burden, it is surprising that only a limited number of studies to date have evaluated the impact of both patient and caregiver characteristics on this construct (Grov et al., 2006; Utne, Miaskowski, Paul, \& Rustøen, 2013) with fewer still focusing on caregivers of colorectal cancer. Further to this, caregiver burden may be exacerbated by objective care-related factors such as hours spent caring (Adelman et al., 2014; van Ryn et al., 2011) and carerelated out-of-pocket (OOP) costs (Longo, Deber, Fitch, Williams, \& D'souza, 2007; van Houtven, Ramsey, Hornbrook, Atienza, \& van Ryn, 2010), yet few studies have examined this aspect of care as a separate predictor of burden beyond the influence of caregiver demographics and patient health status.

Consequently, this study aimed to systematically analyse the key predictors of subjective burden of care in caregivers of colorectal cancer patients in a hierarchical fashion. By first controlling for the influence of (1) caregiver characteristics, we aimed to examine how, (2) patient characteristics (in terms of health-related quality-of-life and clinical characteristics) and (3) objective care-related factors, predict four distinct aspects of caregiver burden.

\section{2 | METHODS}

\section{1 | Participants}

The sample of informal caregivers was derived from a survey of 1,326 colorectal cancer patients (ICD10 C18-20) identified from the National Cancer Registry, Ireland (NCRI) and diagnosed during October 2007-September 2009. Following ethical review and approval from the hospital ethics boards, cancer patients were invited to complete a questionnaire. Exclusion criteria included a poor understanding of English, a history of dementia or other cognitive impairment that would prevent informed consent and being too ill to participate. Of the 495 patients that returned the questionnaire, 228 provided contact details for a family member or friend who had been helping take care of them since their diagnosis. The caregiver postal questionnaire was dispatched during August 2010-March 2011. Up to two reminders were sent to non-respondents at fortnightly intervals. A total of 153 caregivers completed the questionnaire and were included in the analysis with their corresponding patients.

\section{2 | Instruments}

\subsection{1 | Patient questionnaire and clinical information}

Patient characteristics were derived from the patient questionnaire and information held by the NCRI. The patient questionnaire was developed based on relevant literature (e.g. Longo et al., 2007; Yabroff, Warren, Knopf, Davis, \& Brown 2005) and further informed by qualitative discussions with colorectal cancer survivors, patient support groups and health professionals involved in providing care (O'Ceilleachair et al., 2012). In addition to socio-demographic questions and time of diagnosis, patients were asked to indicate whether they had undergone surgery, chemotherapy and/or radiotherapy, and whether they currently had a stoma. Global health status was measured using the EORTC QLQ30. We chose to focus on this element of the QLQ30 rather than the functional and symptom scales given that our primary concern was how overall perceived health-related quality-of-life impacts on caregiver burden. This was based on questions measuring general health and quality-of-life. Responses for global health items ranged from 1 (very poor) to 7 (excellent) and were subsequently transformed into a score between 0 and 100 as recommended (Fayers, 2001). This QLQ30 has shown to have good reliability in other patient populations (Aaronson et al., 1993; Smith, Cocks, Taylor, \& Parry, 2014). Additional clinical information on cancer site (rectal or colon) and stage of diagnosis were taken from NCRI records.

\subsection{2 | Caregiver questionnaire}

The caregiver questionnaire was also developed from literature (e.g. Brouwer et al., 2004; Grov et al., 2006; Nijboer et al., 2000) and qualitative discussions with survivors and their family members (O'Ceilleachair et al., 2012). The instrument included sociodemographic questions pertaining to age, gender, marital status and whether they had children. Additional questions ascertained the caregiver's relationship with the colorectal cancer patient and the existence of any co-morbid conditions. Information was requested on any caring-related OOP costs incurred per week in the last 30 days (under the headings of medicine, household items and 


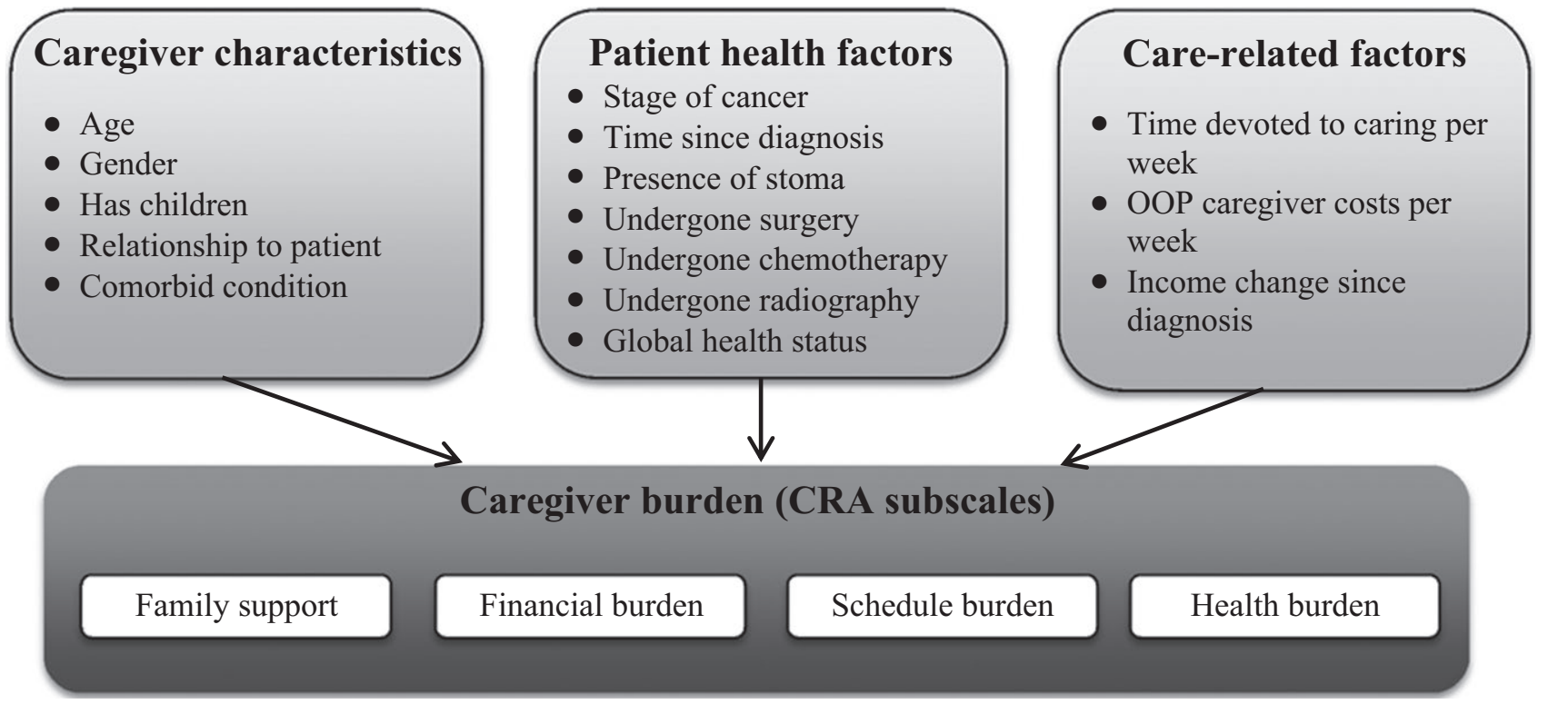

FIGURE 1 An overview of predictor and criterion variables and related blocks included in the four caregiver burden hierarchical multiple regression models

cancer-related items such as home help, private nurse and stoma expenses), and whether the participant experienced a fall, increase, or no change in income since caring for their family member/ friend. Further information was requested on time spent per week (in the last 30 days) on domestic-related caring activities categorised as housework, activities of daily living ( $A D L$ ), instrumental ADL and cancer-specific care (van den Berg \& Spauwen, 2006).

The caregiver reaction assessment (CRA) scale was used to measure caregiver burden (Given et al., 1992). This 24-item multidimensional instrument measures four independent dimensions of burden: (1) impact on schedule, which captures the effects of caregiving on the caregivers' daily activities, (2) impact on finances, which captures the effect of financial strain due to caregiver tasks, (3) impact on health, which measures any perceived deterioration in health from caring and (4) lack of family support, which measure caregivers' perceived lack of support in carrying out caring tasks. One positive dimension is also included in the CRA (impact on caregiver esteem), but this was not considered in this study given our focus on caregiver burden. Participants were asked to indicate their level of agreement with statements relating to their caregiving experience on a 5-point Likert scale ( 1 = strongly disagree, 5 = strongly agree). For the four burden subscales, a higher score represents a greater level of perceived burden. Mean scores are calculated as the average of associated item scores and range from 1.0 to 5.0. The validity and reliability in populations of cancer caregivers has been established previously (Grov et al., 2006; Park et al., 2012). In our sample, individual subscales were found to be reliable based on Cronbach's a coefficients which ranged between .708 and .838 .

\section{3 | Statistical analysis}

Four hierarchical multiple regression models were specified and tested in Statistical Package for Social Sciences (SPSS) 21, one for each of the four measures of caregiver burden. Each model contained three blocks of predictor variables: (1) caregiver characteristics, (2) patient characteristics, (3) care-related factors. Figure 1 illustrates the structure of these models.

Descriptive statistics including means, medians, ranges and SDs were calculated. Prior to the testing of the regression models, preliminary analyses were conducted to ensure no violation of the assumptions of normality, linearity and homoscedasticity (Tabachnick \& Fidell, 2013). Additionally, the correlations among the predictor variables were assessed to ensure multicollinearity was not an issue.

\section{3 | RESULTS}

In total, 153 caregivers completed the questionnaire (response rate = 68\%). Table 1 presents descriptive statistics pertaining to patient characteristics, care-related factors and caregiver characteristics.

\subsection{CRA burden domain scores}

A one-way repeated measured ANOVA was conducted to compare the four burden factors. There was a large, significant difference $(p<.001)$ between the four burden types: Wilks' Lambda $=.37$, $F(3,144)=80.66$, partial eta squared $=.63$. The most negatively affected CRA domain was impact on schedule $(M=3.05, S D=.97)$ which was followed by impact on finances $(M=2.42, S D=.87)$, impact on health $(M=2.34, S D=.73)$ and lack of family support $(M=2.00, S D=.79)$. Results of the Bonferroni post hoc analysis indicated financial burden, schedule burden and health burden were all significantly greater than family support burden. Schedule burden was greater than financial and health burden, while there was no difference between financial and health burden. 
TABLE 1 Demographic characteristics of patients and caregivers

\begin{tabular}{|c|c|c|}
\hline & $n$ & Per cent ${ }^{a}$ \\
\hline \multicolumn{3}{|l|}{ Caregiver characteristics } \\
\hline \multicolumn{3}{|l|}{ Gender } \\
\hline Male & 27 & 18.1 \\
\hline Female & 122 & 81.9 \\
\hline \multicolumn{3}{|l|}{ Children } \\
\hline Have children & 123 & 82.6 \\
\hline Don't have children & 26 & 17.4 \\
\hline \multicolumn{3}{|l|}{ Relationship with patient } \\
\hline Spouse/cohabiting & 108 & 73.0 \\
\hline Other & 40 & 27.0 \\
\hline \multicolumn{3}{|l|}{ Comorbid medical condition(s) } \\
\hline Yes & 40 & 28.4 \\
\hline No & 101 & 71.6 \\
\hline \multicolumn{3}{|l|}{ Patient characteristics } \\
\hline \multicolumn{3}{|l|}{ Gender } \\
\hline Male & 102 & 72.3 \\
\hline Female & 39 & 27.7 \\
\hline \multicolumn{3}{|l|}{ Stage of cancer } \\
\hline Stage 1 or 2 & 53 & 41.7 \\
\hline Stage 3 or 4 & 74 & 58.3 \\
\hline \multicolumn{3}{|l|}{ Presence of a stoma } \\
\hline Currently have a stoma & 36 & 28.6 \\
\hline Don't have a stoma & 90 & 71.4 \\
\hline \multicolumn{3}{|l|}{ Type of treatment undergone ${ }^{b}$} \\
\hline Cancer-directed surgery & 125 & 88.0 \\
\hline Chemotherapy & 43 & 30.3 \\
\hline Radiotherapy & 27 & 19.0 \\
\hline \multicolumn{3}{|l|}{ Time since diagnosis (years) } \\
\hline$<1$ & 51 & 35.2 \\
\hline $1-2$ & 71 & 49.0 \\
\hline More than 2 & 23 & 15.9 \\
\hline $\begin{array}{l}\text { Global health status (possible range: } \\
0-100 \text { ) }\end{array}$ & $\begin{array}{l}67.20 \\
\text { (mean) }\end{array}$ & $21.76(S D)$ \\
\hline \multicolumn{3}{|l|}{ Care-related factors } \\
\hline \multicolumn{3}{|c|}{ Out-of-pocket costs (per week in last 30 days) } \\
\hline None & 68 & 48.2 \\
\hline$€ 1-€ 43.50$ & 36 & 25.5 \\
\hline$€ 44+$ & 37 & 26.2 \\
\hline \multicolumn{3}{|l|}{ Weekly time costs of caring } \\
\hline No extra time & 43 & 32.1 \\
\hline $1-24 \mathrm{~h}$ & 44 & 32.8 \\
\hline $25+h$ & 47 & 35.1 \\
\hline \multicolumn{3}{|l|}{ Income change } \\
\hline No change/increase in income & 93 & 66.0 \\
\hline Income decrease & 48 & 34.0 \\
\hline
\end{tabular}

${ }^{a}$ These figures refer to the percentage of the sample who answered the question.

${ }^{\mathrm{b}}$ Not mutually exclusive; patient may have had one of more of these treatments.

\section{2 | Regression model of schedule burden}

Table 2 summarises the results of the four regression models. Caregiver characteristics (Block 1) explained $6.9 \%$ of variance in levels of schedule burden which was not a statistically significant contribution ( $p=$.18). Patient characteristics were entered in Block 2 and explained an additional $14.4 \%$ of variance $(p=.02)$; while care-related activities (Block 3 ) contributed an additional $12.4 \%$ of variance of schedule burden ( $p=.001$ ) in addition to both caregiver and patient factors. The model as a whole was significant $(p=.001)$ and explained a total of $33.8 \%$ of variance. Three independent variables made a significant contribution to the model: time costs $(\beta=.35, p=.001)$, global health score $(\beta=-.20, p=.039)$ and presence of a stoma $(\beta=.19, p=.040)$.

\section{3 | Regression model of financial burden}

Caregiver characteristics were statistically significant predictors of financial burden ( $p=.011$ ) explaining $13.1 \%$ of variance. Patient characteristics explained an additional $18.4 \%$ of variance $(p=.001$ ), and the care-related factors explained a further significant $5.4 \%$ of financial burden $(p=.051)$. The model as a whole was significant $(p<.001)$ and explained a total of $36.8 \%$ of variance. Three independent variables made a significant contribution: patient global health status $(\beta=-.32, p=.001)$, caregiver age $(\beta=-.26, p=.008)$ and OOP costs $(\beta=.22, p=.033)$

\subsection{Regression model of health burden}

Caregiver characteristics explained $10.9 \%$ of variance in health burden ( $p=.031)$ in block 1 of the model. Patient characteristics explained an additional $12.4 \%$ of variance $(p=.035)$ in block 2 , and carerelated activities explained an additional $8.9 \%$ of variance which was also significant $(p=.008)$. The model as a whole was significant $(p=.001)$ and explained a total of $32.2 \%$ of variance. Four independent variables made a significant contribution predicting health burden: time costs $(\beta=.30, p=.006)$, a comorbid condition for the caregiver $(\beta=.27, p=.004)$, the caregiver having children $(\beta=.23$, $p=.019)$ and patient global health status $(\beta=-.22, p=.025)$.

\section{5 | Regression model of family support burden}

Caregiver characteristics explained $8.3 \%$ of variance in family support burden which was not statistically significant $(p=.101)$. Patient characteristics explained an additional $15.6 \%$ of variance $(p=.009)$ in block 2, while care-related activities explained a further $6.4 \%$ of variance $(p=.038)$. The model as a whole was significant $(p=.001)$ and explained a total of $30.3 \%$ of variance. Four independent variables made a significant contribution to explaining family support burden: time costs involved in caring $(\beta=.28$, $p=.011)$, the patient having undergone chemotherapy $(\beta=.25$, $p=.015)$, patient currently had a stoma $(\beta=.23, p=.015)$ and not being a spouse of the patient $(\beta=-.18, p=.046)$. 
TABLE 2 Hierarchical regression analyses for variables predicting all caregiver burden outcomes

\begin{tabular}{|c|c|c|c|c|c|c|c|c|c|c|c|c|}
\hline \multirow[b]{2}{*}{ Variables } & \multicolumn{3}{|c|}{ Schedule } & \multicolumn{3}{|c|}{ Financial } & \multicolumn{3}{|c|}{ Health } & \multicolumn{3}{|c|}{ Family support } \\
\hline & B & SE & $\beta$ & B & SE & $\beta$ & B & SE & $\beta$ & B & SE & $\beta$ \\
\hline \multicolumn{13}{|c|}{ Block 1: Caregiver characteristics } \\
\hline Age & -.01 & .01 & -.14 & -.02 & .01 & $-.26^{* *}$ & .00 & .01 & -.04 & -.01 & .01 & -.12 \\
\hline Gender & -.13 & .22 & -.05 & .05 & .19 & .02 & .16 & .17 & .08 & -.14 & .18 & -.07 \\
\hline Children & .31 & .24 & .12 & .21 & .21 & .09 & .44 & .18 & $.23^{*}$ & .29 & .20 & .14 \\
\hline Relationship & -.17 & .19 & -.08 & -.05 & .17 & -.02 & -.07 & .15 & -.05 & -.32 & .16 & $-.18^{*}$ \\
\hline Comorbidity & .37 & .19 & .17 & .22 & .17 & .11 & .43 & .15 & $.27^{* *}$ & -.05 & .16 & -.03 \\
\hline$R^{2}$ Change & \multicolumn{3}{|c|}{$.07(p=.18)$} & \multicolumn{3}{|c|}{$.13(p=.011)$} & \multicolumn{3}{|c|}{$.11(p=.031)$} & \multicolumn{3}{|c|}{$.08(p=.101)$} \\
\hline \multicolumn{13}{|c|}{ Block 2: Patient characteristics } \\
\hline $\begin{array}{l}\text { Time since } \\
\text { diagnosis }\end{array}$ & .00 & .00 & -.09 & .00 & .00 & .03 & .00 & .00 & -.04 & .00 & .00 & -.08 \\
\hline Cancer stage & .11 & .18 & .06 & -.26 & .16 & -.15 & .03 & .14 & .02 & -.22 & .15 & -.14 \\
\hline Stoma & .41 & .20 & $.19^{*}$ & .25 & .17 & .13 & .22 & .15 & .13 & .41 & .17 & $.23^{*}$ \\
\hline Surgery & -.12 & .26 & -.04 & -.11 & .23 & -.04 & .02 & .20 & .01 & .00 & .22 & .00 \\
\hline Chemotherapy & -.02 & .21 & -.01 & -.04 & .18 & -.02 & .15 & .16 & .10 & .43 & .18 & $.25^{*}$ \\
\hline Radiotherapy & .05 & .24 & .02 & -.14 & .21 & -.06 & -.08 & .18 & -.04 & -.32 & .20 & -.16 \\
\hline Global Health Score & -.01 & .00 & $-.20^{*}$ & -.01 & .00 & $-.32^{* * *}$ & -.01 & .00 & $-.22^{*}$ & -.01 & .00 & -.15 \\
\hline $\mathrm{R}^{2}$ Change & \multicolumn{3}{|c|}{$.14(p=.02)$} & \multicolumn{3}{|c|}{$.18(p=.001)$} & \multicolumn{3}{|c|}{$.12(p=.035)$} & \multicolumn{3}{|c|}{$.16(p=.009)$} \\
\hline \multicolumn{13}{|c|}{ Block 3: Care-related factors } \\
\hline Time costs & .41 & .12 & $.35^{* * *}$ & -.07 & .11 & -.07 & .26 & .09 & $.30^{* *}$ & .27 & .10 & $.28^{* *}$ \\
\hline OOP costs & .07 & .18 & .06 & .22 & .10 & $.22^{*}$ & .04 & .09 & .05 & -.09 & .10 & -.09 \\
\hline Income change & .04 & .20 & .02 & .27 & .17 & .15 & .04 & .15 & .02 & .18 & .16 & .11 \\
\hline $\mathrm{R}^{2}$ change & \multicolumn{3}{|c|}{$.12(p=.001)$} & \multicolumn{3}{|c|}{$.05(p=.051)$} & \multicolumn{2}{|c|}{$.09(p=.008)$} & & \multicolumn{2}{|c|}{$.06(p=.038)$} & \\
\hline $\mathrm{R}^{2}$ & \multicolumn{2}{|c|}{$.34(p=.001)$} & & \multicolumn{3}{|c|}{$.37(p=.001)$} & \multicolumn{2}{|c|}{$.32(p=.001)$} & & \multicolumn{3}{|c|}{$.30(p=.001)$} \\
\hline
\end{tabular}

Statistical significance: ${ }^{*} p<.05 ;{ }^{* *} p<.01 ;{ }^{* * *} p<.001$.

\section{4 | DISCUSSION}

This study quantified the subjective burden of caring for colorectal cancer patients. By examining how different aspects of burden are influenced separately by caregiver characteristics, patient characteristics and care-related activities, our findings provide a broad picture of colorectal cancer caregiver burden predictors that encompass different elements of the caregiving experience and contribute to growing literature in this area. By systematically analysing these predictors, we can provide a clearer picture of those caregivers most at risk of experiencing burden.

\subsection{Sources of burden}

Consistent with existing research (Adelman et al., 2014; Girgis et al., 2013b) we observed that caring for colorectal cancer patients was associated with perceived burden across a number of domains. Caregivers reported the greatest impact of care on their schedules which highlights the considerable disruption that caring for colorectal cancer patients can cause to daily life. This reflects findings from previous studies on colorectal (Nijboer et al., 2000; Shieh,
Tung, \& Liang, 2012), oral (Chen et al., 2009) and palliative cancer patients (Grov et al., 2006; Park et al., 2012; Utne et al., 2013), yet contradicts other research within colorectal cancer, where health burden (Shieh et al., 2012) and lack of family support (Nijboer et al., 2000) emerged as more onerous sources of burden. These divergent findings may be explained by the differing attitudes, norms, practices and expectations prevalent in a given population which may affect the magnitude of perceived burden between settings. The fact that schedule burden was the most negatively affected domain in our sample suggests that Irish caregivers in particular could benefit from greater support in this respect.

An interesting observation was that financial burden was ranked as highly as health burden suggesting that colorectal caregivers in Ireland may be put under considerable financial strain. Financial burden has been documented within other populations such as palliative cancer (Grov et al., 2006) and oral cancer caregivers (Chen et al., 2009), but is also likely to depend on economic factors such as the nature of social insurance provided or private health insurance held. Ireland has a mixed public-private health care system. All citizens are entitled to treatment within the public system making-at the 
most-modest co-payments for access to services. In addition, a sizable proportion also hold private health insurance $(52 \%$ within our sample), while a notable proportion of low-income individuals, and those aged over 70, are entitled to a medical card which provide free-at-the-point-of-access General Practitioner consultations and public hospital in-patient care and subsidised prescription medications. Previous evidence has shown that Irish cancer patients and caregivers experience a range of costs for cancer-related medical care, in addition to incurring costs for prescription medicines, overthe counter items and dietary supplements (Hanly et al., 2013; Sharp \& Timmons, 2010) and our findings here are compatible with these. Also, a sizable proportion of caregivers take time off work following diagnosis which may not be compensated for monetarily as Carer Allowance is only available to those living with, or providing full-time care, to the care recipient; this may serve to increase the financial burden associated with caregiving and help explain our findings.

The relatively low burden indicated for family support in our sample suggests that most colorectal cancer caregivers in Ireland feel they are adequately supported by family members. This reflects the perceived high level of family cohesion in Ireland and complements the reported support received from family members of patients themselves (O'Ceilleachair et al., 2012). This is a positive finding given that the majority of caregivers in our sample reported that they were the sole caregivers for the care recipient. Nevertheless, the extent of this perceived burden as well as that experienced in the other burden domains differed according to a number of individual predictors, which are elaborated on further below.

\subsection{Caregiver characteristics as predictors of burden}

Caregiver characteristics were the weakest block of predictors of burden overall, yet this varied considerably across the different domains. While having no effect on family support and schedule burden, these characteristics were shown to significantly predict health and financial burden, explaining $11 \%$ and $13 \%$ of explained variance respectively. In particular, the presence of a caregiver comorbid condition was shown to exacerbate health burden, a finding supported by previous research (e.g. Rinaldi et al., 2005). This highlights the additional strain that caring can place on physical well-being for those who already have health complications. Furthermore, we found that caregivers who had children were more likely to report increased health burden suggesting that this group is particularly vulnerable. Conversely, regarding financial burden, we observed that younger caregivers were at an increased risk of strain which is consistent with findings from other populations (Girgis et al., 2013a; Kent et al., 2013; Schneider, Murray, Banerjee, \& Mann, 1999). Also, while caregiver characteristics did not explain family support burden collectively, it should be noted that spousal caregivers were less likely to report a lack of family support suggesting that other family members or friends may suffer more in this respect and could benefit from additional support. Taken together, these findings suggest that specific groups of caregivers of colorectal cancer patients may be identified as having a greater risk of experiencing burden in isolated domains. An appreciation of these risk factors could help anticipate individual caregivers' needs for support at an earlier stage post patient diagnosis.

\section{3 | Patient characteristics as predictors of burden}

The general consensus in the literature is that, across a variety of diseases, patient health is a key driver of the perceived burden of care (Brouwer et al., 2004; Forbes et al., 2007; Kim, Spillers, \& Hall, 2012). Consistent with these findings, the most influential predictor block for all of the caregiver burden domains was patient characteristics, contributing between $14 \%$ and $22 \%$ of variance in the four models. Within this, the patient's own perception of their general health (i.e. global health status) emerged as a significant predictor for all but one aspect of caregiver burden (family support). This suggests that it is patient health-related quality-of-life, rather than more objective disease-related factors, such as stage of cancer (which was not found to be significant in any of the four models), that may dictate the burden of care. It follows that interventions designed to improve patient health-related qualityof-life (Osborn, Demoncada, \& Feuerstein, 2006) may have a key role to play in minimising caregiver burden.

Complementing previous literature which highlighted the negative effect of having a stoma on patients and their caregivers (Jansen et al., 2010; Neuman et al., 2011), the presence of a stoma was also associated with greater schedule burden and lower perceived family support in our sample. Within colorectal cancer patients, coping with a stoma can have numerous detrimental effects such as sleep disruption and difficulty handling daily and social activities (Neuman et al., 2011). Stoma care requires significant time, stamina and commitment from caregivers (Cotrim \& Pereira, 2008; McMullen et al., 2014), thus adding to the disruption caused to caregivers' schedules, over and above their other care tasks. The need for additional support from the family or the healthcare system (perhaps in the form of community nurses) for caregivers of patients with stomas, and those who have received chemotherapy (which emerged as a significant predictor of impact on family support), is also highlighted in our study. Given its invasive nature, chemotherapy can lead to numerous adverse effects and considerable distress among patients (Pettersson, Berterö, Unosson, \& Börjeson, 2014) which can in turn increase burden among caregivers. While our sample generally reported good levels of family support, these findings highlight that those caring for both stoma and chemotherapy patients would further benefit from additional support in carrying out this care.

\subsection{Care-related activities as predictors of burden}

Although patient characteristics accounted for the largest proportion of variance in all caregiver burden models, even after controlling for these and caregiver characteristics, care-related activities 
explained a further significant $5 \%-12 \%$ of the variance. In particular, the time involved in caring emerged as an individual predictor for health, schedule and family support burden which emphasises the role of objective caring factors in predicting perceived burden. In our study, a third of caregivers spent over $25 \mathrm{hr}$ per week undertaking a range of domestic-based care tasks, despite care being undertaken on average $2 \frac{1}{2}$ years post patient diagnosis. In particular, over $2 \mathrm{hr}$ per week were dedicated to personal care activities such as helping the care recipient dress and undress and help in managing pain, administering medicine and changing stoma bags, which may require considerable skill. The range and magnitude of the time dedicated to caring is consistent with previous research in colorectal and lung cancer survivors (van Houtven et al., 2010; van Ryn et al., 2011). While caregiver burden may dissipate somewhat over time (Nijboer et al., 2000) our study revealed that considerable disruption remained, even 2-3 years post diagnosis. This highlights the persistent nature of caregiver tasks and the potential of these to require ongoing changes to a caregiver's routine. Findings also suggest that the time involved in these activities can potentially impact on more than simply caregiver schedule, but also caregiver health. Following from this, interventions targeted at helping caregivers manage their time may be a potential means of mitigating burden. This could, for example, be facilitated through shared care models, in which health professionals in the community (e.g. community nurses) assist in providing certain aspects of care. Our findings suggest that even a small amount of assistance in this respect may help notably reduce caregiver burden.

Beyond the time costs associated with care, another pertinent factor is the financial cost of care. Fifty-two per cent of caregivers in our study incurred some OOP costs in the 30 days prior to questionnaire completion and this factor was found to be a significant predictor of financial burden. Caregiver finances can be affected in a number of ways including excessive OOP costs accumulating over time or a decrease in income due to enforced work absences which may be unpaid. These economic effects are substantial for some cohorts of caregivers, even in countries with a public health service and developed social welfare systems as in Ireland where depression risk was raised threefold in breast, prostate and lung cancer patients reporting increased cancer-related financial stress (Sharp, Carsin, \& Timmons, 2013). Our findings illustrate a need to recognise these costs as separate to the health needs of patients themselves.

\subsection{Strengths and limitations}

This study systematically investigated the role that a variety of patient and caregiver factors had in predicting different aspects of caregiver burden. By separately considering these factors we have provided insight into the diverse and multidimensional nature of the burden of care in an under-researched cancer. However, we acknowledge that the study contains a number of limitations. Given that research suggests burden may change with the trajectory of the illness (Girgis et al., 2013a; Kim et al., 2012) we cannot infer from our cross-sectional design whether a different pattern of results would have emerged had we tracked patients and caregivers over time. Furthermore, our sample of caregivers were nominated by patients themselves and some patients indicated that they had a caregiver but declined to provide contact details. It is possible, therefore, that our sample may not be representative of all those caring for colorectal cancer patients in Ireland. In addition, seventy of the nominated caregivers did not respond to the survey. However, of these, the distributions of caregiver gender and relationship with the patient did not differ significantly between responders and non-responders. While the limited size does not preclude the results being generalisable, larger population-based studies would also be warranted.

The caregiver survey took place approximately 1-2 years after the patient survey when patient health may have changed. While this is a limitation of the study design, the fact that patient healthrelated quality-of-life was a strong predictor of burden even after this elapsed time period is in itself an interesting finding. This suggests that individual differences in patient perceived health-related quality-of-life may persist over time and continue to impact on many aspects of caregiver burden. It is likely that had there been a shorter interval between caregiver and patient questionnaire completion, the predictive value of patient health on burden may have been even stronger.

Like any study, we could not consider all possible predictors so further research could be undertaken to broaden the scope of factors considered. In particular, future research could further explore the role that specific patient symptoms, side effects and functional limitations (many of which predict health-related quality-of-life) may play in influencing burden. More detailed analysis to investigate how specific aspects of financial and time costs predict this burden would also be of value.

\section{5 | CONCLUSION}

As the cancer care model transitions from inpatient-led to outpatientled care, understanding and addressing the needs of informal caregivers will become increasingly important. The extent to which caregivers feel burdened by caring is determined by many distinct and diverse factors. In this study, patient health-related factors were the most influential in determining caregiver burden; however, care-related factors also emerged as significant contributors for all burden domains. Caregiver characteristics were less important, with the exception of predicting caregiver health and financial burden. By revealing groups potentially vulnerable to a higher burden, these findings may potentially assist in the development of a "screening tool" so that those most at risk of high burden may be identified. This may then pave the way for the development of focused interventions to better support colorectal cancer caregivers who are at greatest risk and hence reduce their perceived burden. 


\section{ACKNOWLEDGEMENTS}

Data collection for this study was funded by the Health Research Board (SA/2004/1). We are grateful to the caregiver and colorectal cancer survivors who took part in the surveys, the clinicians and nurses who supported and facilitated the fieldwork, and Alan O'Ceilleachair who undertook the patient survey and identified the caregivers. We also thank the clerical support team at the National Cancer Registry who aided with survey administration and data input.

\section{REFERENCES}

Aaronson, N. K., Ahmedzai, S., Bergman, B., Bullinger, M., Cull, A., Duez, N. J., ... \& Kaasa, S. (1993). The European organization for research and treatment of cancer QLQ-C30: A quality-of-life instrument for use in international clinical trials in oncology. Journal of the National Cancer Institute, 85, 365-376.

Adelman, R. D., Tmanova, L. L., Delgado, D., Dion, S., \& Lachs, M. S. (2014). Caregiver burden: A clinical review. JAMA, 311, 1052-1060.

Applebaum, A. J., \& Breitbart, W. (2013). Care for the cancer caregiver: A systematic review. Palliative and Supportive Care, 11, 231-252.

Arndt, V., Merx, H., Stegmaier, C., Ziegler, H., \& Brenner, H. (2006). Restrictions in quality of life in colorectal cancer patients over three years after diagnosis: A population based study. European Journal of Cancer, 42, 1848-1857.

Bray, F., Ren, J. S., Masuyer, E., \& Ferlay, J. (2013). Global estimates of cancer prevalence for 27 sites in the adult population in 2008. International Journal of Cancer, 132, 1133-1145.

Brouwer, W. B., Van Exel, N., van de Berg, B., Dinant, H. J., Koopmanschap, M. A., \& van den Bos, G. A. (2004). Burden of caregiving: Evidence of objective burden, subjective burden, and quality of life impacts on informal caregivers of patients with rheumatoid arthritis. Arthritis and Rheumatism, 51, 570-577.

O'Ceilleachair, A., Costello, L., Finn, C., Timmons, A., Fitzpatrick, P., Kapur, K., ... \& Sharp, L. (2012). Inter-relationships between the economic and emotional consequences of colorectal cancer for patients and their families: A qualitative study. BMC Gastroenterology, 12, 62. 230X-1262.

Chen, S. C., Tsai, M. C., Liu, C. L., Yu, W. P., Liao, C. T., \& Chang, J. T. C. (2009). Support needs of patients with oral cancer and burden to their family caregivers. Cancer Nursing, 32, 473-481.

Cotrim, H., \& Pereira, G. (2008). Impact of colorectal cancer on patient and family: Implications for care. European Journal of Oncology Nursing, 12, 217-226.

Fayers, P. M. (2001). Interpreting quality of life data: Population-based reference data for the EORTC QLQ-C30. European Journal of Cancer, 37, 1331-1334.

Forbes, A., While, A., \& Mathes, L. (2007). Informal carer activities, carer burden and health status in multiple sclerosis. Clinical Rehabilitation, 21, 563-575.

Girgis, A., Lambert, S. D., McElduff, P., Bonevski, B., Lecathelinais, C., Boyes, A., \& Stacey, F. (2013a). Some things change, some things stay the same: A longitudinal analysis of cancer caregivers' unmet supportive care needs. Psychooncology, 22, 1557-1564.

Girgis, A., Lambert, S., Johnson, C., Waller, A., \& Currow, D. (2013b). Physical, psychosocial, relationship, and economic burden of caring for people with cancer: A review. Journal of Oncology Practice/American Society of Clinical Oncology, 9, 197-202.

Given, C. W., Given, B., Stommel, M., Collins, C., King, S., \& Franklin, S. (1992). The caregiver reaction assessment (CRA) for caregivers to persons with chronic physical and mental impairments. Research in Nursing and Health, 15, 271-283.
Goren, A., Gilloteau, I., Lees, M., \& daCosta DiBonaventura, M. (2014). Quantifying the burden of informal caregiving for patients with cancer in Europe. Supportive Care in Cancer, 22, 1637-1646.

Grov, E. K., Fosså, S. D., Sørebø, Ø., \& Dahl, A. A. (2006). Primary caregivers of cancer patients in the palliative phase: A path analysis of variables influencing their burden. Social Science and Medicine, 63, 2429-2439.

Hanly, P., Céilleachair, A. Ó., Skally, M., O'Leary, E., Kapur, K., Fitzpatrick, P., ... \& Sharp, L. (2013). How much does it cost to care for survivors of colorectal cancer? Caregiver's time, travel and out-of-pocket costs. Supportive Care in Cancer, 21(9), 2583-2592.

Hanly, P., Maguire, R., Hyland, P., \& Sharp, L. (2015). Examining the role of subjective and objective burden in carer health-related quality of life: the case of colorectal cancer. Supportive Care in Cancer, 23(7), 1941-1949.

Jansen, L., Koch, L., Brenner, H., \& Arndt, V. (2010). Quality of life among long-term ( $\geq 5$ years) colorectal cancer survivors-systematic review. European Journal of Cancer, 46, 2879-2888.

Kent, E. E., Forsythe, L. P., Yabroff, K. R., Weaver, K. E., Moor, J. S., Rodriguez, J. L., \& Rowland, J. H. (2013). Are survivors who report cancer-related financial problems more likely to forgo or delay medical care? Cancer, 119, 3710-3717.

Kim, Y., Spillers, R. L., \& Hall, D. L. (2012). Quality of life of family caregivers 5 years after a relative's cancer diagnosis: Follow-up of the national quality of life survey for caregivers. Psychooncology, 21, 273-281.

Longo, C. J., Deber, R., Fitch, M., Williams, A. P., \& D'souza, D. (2007). An examination of cancer patients' monthly 'out-of-pocket' costs in Ontario, Canada. European Journal of Cancer Care, 16, 500-507.

McMullen, C. K., Schneider, J., Altschuler, A., Grant, M., Hornbrook, M. C., Liljestrand, P., \& Krouse, R. S. (2014). Caregivers as healthcare managers: health management activities, needs, and caregiving relationships for colorectal cancer survivors with ostomies. Supportive Care in Cancer, 22, 2401-2408.

Neuman, H. B., Patil, S., Fuzesi, S., Wong, W. D., Weiser, M. R., Guillem, J. G., ... \& Temple, L. K. (2011). Impact of a temporary stoma on the quality of life of rectal cancer patients undergoing treatment. Annals of Surgical Oncology, 18, 1397-1403.

Nijboer, C., Triemstra, M., Tempelaar, R., Mulder, M., Sanderman, R., \& van den Bos, G. A. (2000). Patterns of caregiver experiences among partners of cancer patients. The Gerontologist, 40, 738-746.

Ohlsson-Nevo, E., Andershed, B., Nilsson, U., \& Anderzén-Carlsson, A. (2012). Life is back to normal and yet not - partners' and patient's experiences of life of the first year after colorectal cancer surgery. Journal of Clinical Nursing, 21, 555-563.

Olson, R. E. (2012). Is cancer care dependant on informal carers? Australian Health Review, 36, 254-257.

Osborn, R. L., Demoncada, A. C., \& Feuerstein, M. (2006). Psychosocial interventions for depression, anxiety, and quality of life in cancer survivors: Meta-analyses. International Journal of Psychiatry in Medicine, 36, 13-34.

Park, C. H., Shin, D. W., Choi, J. Y., Kang, J., Baek, Y. J., Mo, H. N., ... \& Park, S. (2012). Determinants of the burden and positivity of family caregivers of terminally ill cancer patients in Korea. Psychooncology, 21, 282-290.

Pettersson, G., Berterö, C., Unosson, M., \& Börjeson, S. (2014). Symptom prevalence, frequency, severity, and distress during chemotherapy for patients with colorectal cancer. Supportive Care in Cancer, 22, 11711179.

Rinaldi, P., Spazzafumo, L., Mastriforti, R., Mattioli, P., Marvardi, M., Polidori, M. C., ... \& Capurso, A. (2005). Predictors of high level of burden and distress in caregivers of demented patients: results of an Italian multicenter study. International Journal of Geriatric Psychiatry, 20, 168-174.

Rodakowski, J., Skidmore, E. R., Rogers, J. C., \& Schulz, R. (2012). Role of social support in predicting caregiver burden. Archives of Physical Medicine and Rehabilitation, 93, 2229-2236. 
Romito, F., Goldzweig, G., Cormio, C., Hagedoorn, M., \& Andersen, B. L. (2013). Informal caregiving for cancer patients. Cancer, 119(Suppl. 11), 2160-2169.

Sautter, J. M., Tulsky, J. A., Johnson, K. S., Olsen, M. K., Burton-Chase, A. M., Hoff Lindquist, J., ... \& Steinhauser, K. E. (2014). Caregiver experience during advanced chronic illness and last year of life. Journal of the American Geriatrics Society, 62, 1082-1090.

Schneider, J., Murray, J., Banerjee, S., \& Mann, A. (1999). Eurocare: a crossnational study of co-resident spouse carers for people with Alzheimer's disease: I-Factors associated with carer burden. International Journal of Geriatric Psychiatry, 14, 651-661.

Sharp, L., Carsin, A. E., \& Timmons, A. (2013). Associations between cancerrelated financial stress and strain and psychological well-being among individuals living with cancer. Psychooncology, 22, 745-755.

Sharp, L., \& Timmons, A. (2010). The financial impact of a cancer diagnosis. Cork: National Cancer Registry.

Shieh, S. C., Tung, H. S., \& Liang, S. Y. (2012). Social support as influencing primary family caregiver burden in Taiwanese patients with colorectal cancer. Journal of Nursing Scholarship, 44, 223-231.

Sjovall, K., Attner, B., Englund, M., Lithman, T., Noreen, D., Gunnars, B., ... \& Petersson, I. F. (2011). Experiences of living with advanced colorectal cancer from two perspectives - inside and outside. European Journal of Oncology Nursing, 15, 390-397.

Sjovall, K., Gunnars, B., Olsson, H., \& Thomé, B. (2012). Sickness absence among cancer patients in the pre-diagnostic and the post-diagnostic phases of five common forms of cancer. Supportive Care in Cancer, 20, 741-747.

Smith, A. B., Cocks, K., Taylor, M., \& Parry, D. (2014). Most domains of the European Organisation for Research and Treatment of Cancer Quality of Life Questionnaire C30 are reliable. Journal of Clinical Epidemiology, 67, 952-957.

Tabachnick, B., \& Fidell, L. S.. (2013). Using multivariate statistics (6th edn). New York: Pearson.
Utne, I., Miaskowski, C., Paul, S. M., \& Rustøen, T. (2013). Association between hope and burden reported by family caregivers of patients with advanced cancer. Supportive Care in Cancer, 21, 2527-2535.

van den Berg, B., \& Spauwen, P. (2006). Measurement of informal care: An empirical study into the valid measurement of time spent on informal caregiving. Health Economics, 15, 447-460.

van Houtven, C. H., Ramsey, S. D., Hornbrook, M. C., Atienza, A. A., \& van Ryn, M. (2010). Economic burden for informal caregivers of lung and colorectal cancer patients. The Oncologist, 15, 883-893.

van Ryn, M., Sanders, S., Kahn, K., van Houtven, C., Griffin, J. M., Martin, M., ... \& Rowland, J. (2011). Objective burden, resources, and other stressors among informal cancer caregivers: A hidden quality issue? PsychoOncology, 20, 44-52.

Yabroff, K. R., \& Kim, Y. (2009). Time costs associated with informal caregiving for cancer survivors. Cancer, 115(18 Suppl.), 4362-4373.

Yabroff, K. R., Warren, J. L., Knopf, K., Davis, W. W., \& Brown, M. L. (2005). Estimating patient time costs associated with colorectal cancer care. Medical Care, 43, 640-648.

Yoon, S. J., Kim, J. S., Jung, J. G., Kim, S. S., \& Kim, S. (2014). Modifiable factors associated with caregiver burden among family caregivers of terminally ill Korean cancer patients. Supportive Care in Cancer, 22, 1243-1250.

How to cite this article: Maguire R, Hanly P, Hyland P, Sharp L. Understanding burden in caregivers of colorectal cancer survivors: what role do patient and caregiver factors play? Eur J Cancer Care. 2018;27:e12527. https://doi.org/10.1111/ ecc.12527 